\title{
Uso de fotoprotetores em caminhoneiros
}

\author{
Impacts of extrinsic use of photoprotectors in trucks \\ Uso de fotoprotectores en camiones
}

Recebido: 10/06/2021 | Revisado: 16/06/2021 | Aceito: 18/06/2021 | Publicado: 29/06/2021

\author{
Laysa Michaelle Oliveira Santos \\ ORCID: https://orcid.org/0000-0002-4009-7519 \\ Faculdade Independente do Nordeste, Brasil \\ E-mail: laysamichaelle@gmail.com \\ Jeane Rocha Santos \\ ORCID: https://orcid.org/0000-0002-1398-3638 \\ Faculdade Independente do Nordeste, Brasil \\ E-mail: Jeane@fainor.com.br \\ Ana Rachel Moreno Caetité \\ ORCID: https://orcid.org/0000-0003-1329-7440 \\ Faculdade Independente do Nordeste, Brasil \\ E-mail: Anarachel@ fainor.com.br \\ Celina Coqueiro Cunha \\ ORCID: https://orcid.org/0000-0003-0654-2057 \\ Faculdade Independente do Nordeste, Brasil \\ E-mail: Celinacoqueiro@ outlook.com
}

\begin{abstract}
Resumo
Introdução: Alterações na pele e câncer de pele resultantes da exposição ao sol representam um problema de saúde global, e seu controle, prevenção e promoção são de responsabilidade do setor de Saúde Pública e de todos. A proteção da luz é essencial, pois os danos causados pelo sol são cumulativos e podem aumentar o risco de câncer de pele e distúrbios da pele. Embora a exposição à luz solar tenha efeitos benéficos, como sintomas de bem-estar, produção. Objetivo: Analisar a eficácia de proteção do uso de fotoprotetores em caminhoneiros, elencando as doenças desencadeadas pela exposição solar nesses profissionais. Método: Pesquisa descritiva de natureza qualitativa e e revisão bibliográfica. Resultados: A investigação demostrou que muitos dos caminhoneiros não fazem uso de fotoprotetores, o que os expõe ao risco de desenvolver doenças de pele como o câncer de pele. Conclusão: Há uma necessidade de sensibilizar os profissionais caminhoneiros sobre a importância do uso de fotoprotetores.
\end{abstract}

Palavras-chave: Fotoproteção; Carcinogênese; Trabalhadores caminhoneiros.

\begin{abstract}
Introduction: Skin changes and skin cancer resulting from sun exposure represent a global health problem, and its control, prevention and promotion are the responsibility of the Public Health sector and everyone. Protection from light is essential as sun damage is cumulative and can increase the risk of skin cancer and skin disorders. Although exposure to sunlight has beneficial effects, such as symptoms of well-being, production. Objective: To analyze the protection effectiveness of the use of photoprotectors in truck drivers, listing the diseases triggered by sun exposure in these professionals. Method: Descriptive research of qualitative nature and bibliographical review. Results: The investigation showed that many of the truck drivers do not use sunscreens, which exposes them to the risk of developing skin diseases such as skin cancer. Conclusion: There is a need to sensitize truck drivers about the importance of using sunscreens.
\end{abstract}

Keywords: Photoprotection; Carcinogenesis; Truck drivers.

\section{Resumen}

Introducción: Las alteraciones cutáneas y el cáncer de piel derivados de la exposición solar representan un problema de salud global, y su control, prevención y promoción son responsabilidad del sector Salud Pública y de todos. La protección contra la luz es esencial, ya que el daño solar es acumulativo y puede aumentar el riesgo de cáncer de piel y trastornos de la piel. Aunque la exposición a la luz solar tiene efectos beneficiosos como síntomas de bienestar, producción. Objetivo: Analizar la efectividad protectora del uso de filtros solares en camioneros, enumerando las enfermedades desencadenadas por la exposición solar en estos profesionales. Método: Investigación descriptiva de carácter cualitativo y revisión bibliográfica. Resultados: La investigación mostró que muchos de los camioneros no usan protectores solares, lo que los expone al riesgo de desarrollar enfermedades de la piel como cáncer de piel. Conclusión: Es necesario sensibilizar a los conductores de camiones sobre la importancia del uso de protectores solares.

Palabras clave: Fotoprotección; Carcinogénesis; Camioneros. 


\section{Introdução}

A maioria dos efeitos biológicos da luz solar na pele humana se deve à radiação ultravioleta, que é filtrada através de camadas atmosféricas, permitindo a passagem de um espectro oscilante (Cortez, 2016). Os efeitos nocivos do sol são cumulativos e irreversíveis. Alguns são imediatos, como o aparecimento de eritema, pigmentação ou queimadura. Outros ocorrem tardiamente, como envelhecimento da pele ou aumento do risco de carcinogênese (Neves, 2006).

Pode ocorrer ainda, distúrbios de fotosensibilidade pela supressão do sistema imunológico. Existem várias doenças agravadas pela exposição solar, a exemplo do lúpus, porfiria ou xeroderma pigmentoso, que afetam caracteristicamente as crianças (Oliveira, 2011).

A intensa exposição ao sol durante as duas primeiras décadas de vida tem sido especialmente relacionada ao fotoenvelhecimento da pele e à formação de câncer de pele. O câncer cutâneo é o tipo de câncer mais frequente no mundo e o melanoma é o que mais cresce, de acordo com as últimas publicações da Organização Mundial da Saúde (Gordon et.al; 2015).

Foi demonstrada uma intensa correlação entre queimaduras na infância e risco de melanoma. Também foi documentada uma relação entre melanoma e exposição intermitente à exposição solar. Existe também uma associação entre a exposição solar na infância e o número de nevo melanocítico adquirido, cujo alto número também é considerado fator de risco para melanoma (WANG, 2016).

Nesse contexto, os Fotoprotetores são aquelas substâncias que absorvem e filtram a radiação UV (impedindo sua penetração na epiderme e derme), dispersam e refletem a radiação. Alguns fotoprotetores atuais também têm a capacidade de inativar os radicais livres produzidos e, portanto, impedir ou reparar os danos causados pela radiação solar. Seu uso é essencial, uma vez que constituem uma medida fundamental de proteção contra a radiação ultravioleta. Eles demonstraram ter efeitos positivos na diminuição dos sinais de envelhecimento e na incidência de câncer de pele (Silva, 2016).

Hoje no Brasil, inúmeras pessoas ainda desconhecem a importância do uso dos protetores solares na prevenção de doençãs e do envelhecimentos precoce. Dentre esses individuos, se destacam o profissional, que se caracterizam como uma profissão que submete a exposição solar, visto que grande parte desses individuos passam horas dirigindo, recebendo luz direta através das janelas dos veículos. Grande parte desses profissionais no Brasil não dispoem de conhecimentos acerca da importância dos fotoprotetores o que ocasiona o desenvolvimento de envelhecimento precoce e cancer de pele (Silva, et.al; 2016).

A pesquisa, justifica-se pela relevância de compreender que a exposição solar, é essêncial para a vida, porém em certas circunstâncias pode causar efeitos danos a pele, originando vários quadros patológicos, entre os quais queimaduras solares, fotoenvelhecimento, fotodermatose e imunossupressão. Tomar sol com o único objetivo de ostentar um corpo bronzeado não é aconselhável, pois os raios ultravioleta podem acabar com sérios problemas de saúde. Para aproveitar os raios solares, ser riscos é importante conhecer conceitos básicos em foto-proteção. Para as pesoas que necesitam.

trabalhar diariamente exposto ao sol, se torna de suma importãncia fazer uso de fotoprotetores. Frente ao exposto, esta pesquisa tem como objetivo geral analisar a eficácia de proteção do uso de fotoprotetores em caminhoneiros, elencando as doenças desencadeadas pela exposição solar nesses profissionais; e como objetivos específicos: Demostrar a incidência do uso de fotoprotetores em caminhoneiros; Avaliar a constituição da pele dos caminhoneiros; Analisar a relação entre o não uso de fotoprotetores e envelhecimento precoce. Analisar o conhecimento dos profissionais caminhoneiros acerca das doenças relacionadas a exposição solar.

\section{Metodologia}

Trata-se de uma pesquisa descritiva de natureza qualitativa e revisão bilbiográfica. Na pesquisa qualitativa, o cientista é ao mesmo tempo o sujeito e o objeto de suas pesquisas. O desenvolvimento da pesquisa é imprevisível. O conhecimento do 
pesquisador é parcial e limitado (Deslauriers, 1991).

Para identificação das fontes, utilizou-se um recorte de 05 anos, sendo coletados artigos publicados no período de 20105 a 2020. Foi utilizado filtros nas buscas, procedendo da seguinte maneira: data de publicação dos artigos; descritores, Fotoproteção, Carcinogênese, Trabalhadores caminhoneiros.po de literatura. Os estudos duplicados foram analisados e excluidos. Após a leitura dos artigos, realizou-se fichamento com ficha catalografica, contendo infromações como: título, autores, periódico, país, idioma, ano de publicação, objetivos, metodologia, resultados da pesquisa, destacando os estudos com abordagem sobre o uso de fotoprotetores em caminhoneiros

Utilizou-se a busca Booleana, combinando as palavras-chaves, Fotoproteção, Carcinogênese, Trabalhadores caminhoneiros, usando o operador AND. Não foi utilizado os nomes de autores na busca. Além disso, foi inserido filtros de busca nas plataformas online, como data de publicação (2010 a 2020), artigos publicados na língua portuguesa, inglesa e espanhol. Após o cruzamento, será realizada a leitura dos resumos com o objetivo de averiguar a afinidade dos artigos com a temática do estudo, tendo como base a questão norteadora, em como excluir as duplicidades.

A análise dos dados, será realizada a partir da leitura dos artigos selecionados. Os dados extraídos serão organizados em planilhas em ordem numérica crescente, no programa Microsoft Office Excel 2010, em colunas que contenham informações sobre: título, autor(es), ano de publicação, objetivo da pesquisa, método, principais resultados e conclusão.

Esse estudo obedece aos aspectos éticos de acordo com a Lei $\mathrm{n}^{\circ}$ 9.610, de 19 de fevereiro de 1998, que dispõe sobre direitos autorais. Por se tratar de pesquisa de revisão de literatura não será necessária a submissão ao comitê de ética.

\section{Resultados e Discussão}

Os achados desta investigação evidenciaram os seguintes resultados:

A exposição prolongada ao sol pode causar sérios problemas de pele, por isso é muito importante conhecer os riscos relacionados à radiação solar, bem como o uso adequado do protetor solar, em um momento de altas temperaturas, sendo as radiações mais perigosas (Costa, 2017).

É importante ressaltar que as pessoas que estão mais expostas ao sol apresentam sinais precoces de envelhecimento de sua pele, por isso é muito importante que na hora de escolher um protetor solar, eles devem ter filtros tanto para radiação UVA quanto UVB. Onde o fator de proteção solar (FPS) é um elemento a ter em consideração (Cabral, 2013).

Ressalta-se que os tumores de pele são os mais comuns de todos os cânceres e costumam ser divididos em dois tipos: melanomas e os chamados tumores não melanomatosos, compostos por carcinomas espinocelulares e basocelulares (Wang, 2016).

O segundo tipo de neoplasias é dez vezes mais frequente que os melanomas e de malignidade relativamente baixa, mas se não forem detectados nos estágios iniciais podem originar, após o tratamento, cicatrizes e deformações de grande repercussão estética e psicológica (Godinho, 2017). O melanoma, por outro lado, é um dos cânceres com maior mortalidade relativa devido à sua alta propensão a metastatizar precocemente e também afeta uma população relativamente jovem, o que o torna um dos tumores malignos mais importantes em termos de anos de vida potenciais perdidos a cada morte (Albert, 1990).

Sabe-se que pessoas com pele clara, sardas, cabelos loiros ou ruivos e que sofrem de queimaduras solares com facilidade, são geneticamente predispostas a sofrer de tumores de pele devido à sua menor capacidade de desenvolver um bronzeado protetor na pele (Souza, 2011). Uma história familiar direta de melanoma é decisiva na probabilidade de sofrer de câncer. Além disso, a propensão para o desenvolvimento de certas doenças hereditárias da pele, como xeroderma pigmentosa ou síndrome do nevo displásico familiar, causam um grande aumento no risco de tumores de pele, especialmente câncer, em comparação com a população em geral (Silva, 2014). 
Como já mencionado, a exposição aos raios ultravioleta é o fator de risco externo mais importante para o desenvolvimento de tumores cutâneos, no caso do melanoma, estima-se que na Europa entre 54\% e 80\% desses tumores sejam decorrentes da exposição à radiação ultravioleta, embora sua relação com tumores não melanomatosos também esteja bem estabelecida (Gallagher, 1995).

Nessa otica, os cremes solares podem ser uma boa ferramenta de proteção contra os raios ultravioleta, mas por si só não garantem a prevenção do câncer de pele se seu uso não estiver vinculado à redução da exposição solar (Balogh, 2011). Na verdade, os cremes surgiram no mercado para permitir uma maior permanência ao sol e usados para esse fim não seriam úteis na profilaxia de tumores cutâneos. Devem, portanto, ser considerados apenas como um fator adjuvante para fotoproteção (Cruz, 2016).

O poder de filtragem de um creme é medido por meio do fator de proteção solar (FPS) que ele possui, e que traduz a capacidade do produto em retardar o eritema solar, efeito causado pelos raios UVB, portanto não valoriza a radiação UVA (Labs, 2018). É expressa por um número que indica o múltiplo de tempo ao qual a pele protegida deve ficar exposta, para atingir o mesmo efeito eritematoso que seria obtido se nenhuma proteção tivesse sido aplicada. Ou seja, se o eritema surgisse em determinada pele após 20 minutos de exposição ao sol, a mesma pele após a aplicação de protetor solar com FPS 6 ficaria eritematosa após 120 minutos (Tuorkey, 2015). No entanto, o FPS não tem uma relação linear com a proteção que fornece, mas sim logarítmica, de modo que, em números elevados, aumentos no FPS não se traduzem em aumentos proporcionais na fotoproteção (Cruz, 2016).

O uso de cremes solares demonstrou reduzir o efeito imunossupressor causado pela radiação UV, medido como a capacidade de inibir a hipersensibilidade de contato com a pele (tipo IV) (INCA, 2017).

O fator de imunoproteção do creme não está diretamente relacionado ao fator de proteção solar (prevenção do eritema), mas sim à sua maior ou menor capacidade de bloquear a passagem dos raios UVA (Wolf, 2003). Por outro lado, o uso em "condições normais" de cremes de proteção solar não parece levar a uma diminuição significativa na produção de vitamina D na pele, embora haja uma tendência crescente de viver em espaços internos não ensolarados, acrescentou. o uso de roupas que cubram toda a pele e o uso somado à fotoproteção exaustiva podem, em conjunto, levar à produção dérmica insuficiente de vitamina D (Wang, 2016).

É reconhecido que para a fotoproteção adequada é necessário o uso de um creme com FPS 15 ou superior, embora o fator ideal dependa em cada caso tanto das características da pele quanto do tempo de exposição esperado e de outros fatores ambientais já descritos (Pasini, 2019). Dessa forma, sabe-se que os profssionais caminhoneiros se expoe há grandes jornadas diárias de trabalho, e inevitavelmente ficam diretamente expostos a luz sola. A melhor maneira de diminuir o risco de alterações na pele é protegendo-a do sol. Isso inclui o uso de filtros ou protetores solares e outras medidas de proteção (Mancebo, 2014).

É importante evitar a exposição ao sol, principalmente durante as horas de 10h às 16h, quando os raios ultravioleta são mais fortes. Também é importante lembrar que quanto maior a altitude, mais rápido sua pele queima com a exposição ao sol. Deve-se usar proteção solar, mesmo em dias nublados. Nuvens e neblina não protegem do sol e podem até intensificar os raios UVB (Uorkey, 2015).

\section{Conclusão}

A pesquisa realizada permitiu identificar que o principal fator associado ao processo de fotoenvelhecimento e doenças de pele está relacionado à exposição excessiva e desprotegida aos raios ultravioletas do sol. Eficácia de proteção do uso de fotoprotetores em caminhoneiros, elencando as doenças desencadeadas pela exposição solar nesses profissionais. 
Os achados dessa investigação demostraram que muitos caminhoneiros não fazem o uso de fotoprotetores o que os expõe ao risco de desenvolver doenças como o câncer de pele, imprescindível para minimizar os efeitos do envelhecimento precoce em individuos que não fazem o uso de fotoprotetores.

$\mathrm{O}$ contexto geral dessa pesquisa reflete a necessidade de conscientizar os profissioanis caminhoneiros sobre a importância do uso de fotoprotetores, juntamente com o uso de acessórios que auxiliam na proteção, no sentido de minimizar os danos relacionados ao fotoenvelhecimento e doenças de pele.

Esse estudo contribuiu para demostrar o uso de fotoprotetores em caminhonerios e o conhecimento desses profissionais acerca da importancia de se proteger da exposição solar.

Recomenda-se que novos estudos sejam desenvolvidos, a fim de aprofundar o conhecimento sobre o uso de fotoprotetores em caminhoneiros, visto que se trata de umtema de grande relevância na atualidade.

\section{Referências}

Albert, V. A., Koh, H. K., Geller, A. C., Miller, D. R., Prout, M. N., \& Lew, R. A. Years of potential life lost: another indicator of the impact of cutaneous malignant melanoma on society. J Am Acad Dermatol. (1990),23:308-310.

Balogh, T. S. Velasco, M. V. R., Pedriali, C. A., Kaneko, T. M., \& Baby, A. R. (2011). Proteção à radiação ultravioleta: recursos disponíveis na atualidade em fotoproteção. An Bras Dermatol, 86(4), 732-42.

Cabral, L. D. S., Pereira, S. O., \& Partata, A. K. Filtros solares e fotoprotetores - uma revisão. Infarma, 25(2)

Cortez, D. A. G., Machado E. S, Vermelho, S. C. S. D., Teixeira, J. J. V., \& Cortez, L. E. R. O conhecimento e a utilização de filtro solar por profissionais da beleza. Ciência \& Saúde Coletiva. 2016, 21(7): 2267-74.

Costa, R. M. Novos avanços tecnológicos na fotoproteção. 2017. 59 f. Dissertação (Mestrado em Ciências Farmacêuticas) - Universidade Lusófona de Humanidades e Tecnologias, Escola de Ciências e Tecnologia da Saúde, Lisboa 2017.

Cruz, V. M. F. R., Acosta-Avalos, D., \& Barja, P. R. (2016). Estudo Da Fotoestabilidade De Protetores Solares Por Espectroscopia Fotoacústica.

Deslaurlers, j.-P. \&. Brassard, M.-J. (1989). Pouvoir habiter. Chicoutimí: Universidade do Québec em Chicoutim

Gallagher, R., Hill, G., Bajdik, C. D., et al. Sunlight exposure, pigmentary factors, and risk of nonmelanocytic skin cancer. I. Basal cell carcinoma. Arch Dermatol. (1995),131:157-163.

Godinho, M. M., Hossy, B. H., Niemeyer-Corbellini, J. P., \& Silva, M. R. Perfil dos filtros solares utilizados nos fotoprotetores no Brasil. Sociedade Brasileira de Dermatologia Brasil. 2017. 9(3), 243-246

Gil, A. C. Como elaborar projetos de pesquisa. Atlas, (2002).

Guirro, E. C. O. \& Guirro. R. R. J., Fisioterapia dermato-funcional: fundamentos, recursos, patologias. (3a ed.), Manole, (2004).

INCA - Instituto Nacional do Câncer - Dados câncer de pele, 2017.

Labs, T. C., Fernandes Vivian, R. H., Silva, T. O., \& Costa, M. C. D. Melanoma e o uso de filtro solar como forma de prevenção. Revista Terra e Cultura. 2018. 1(1), 236-240.

Mancebo S. E., Hu J. Y., \& Wang S. Q. Sunscreens: a review of health benefits, regulations, and controversies. Dermatol Clin. (2014) 32(3):427-38.

Neves, C. E. B. Estimativa da Composição Corporal por Espectroscopia de Impedância Bio-Elétrica Bipolar em Indivíduos com Sobrepeso e Obesidade", Tese D.Sc., Universidade Federal do Rio de Janeiro.

Oliveira, L. F. Análise morfológica e imunológica da pele, de acordo com as características epidemiológicas de idosos autopsiados (2011).

Pasini, M. V. S. Design de dispositivo dosador de protetor solar. 2019. 137 f. Monografia (Bacharelado em Design). Faculdade de Arquitetura, Universidade Federal do Rio Grande do Sul, Porto Alegre, RS, 2019.

Reenlee, R. T., Hill-Harmon, M. B., Murray, T., \& Thun, M. Cancer statistics (2001). CA Cancer J Clin..

Silva, V. P., Paiva, T. M. A., Sousa, T. A. A., \& Marques, R. C. P. Câncer de pele: uso de medidas preventivas e perfil demográfico de um grupo de risco na cidade de Mossoró/RN. Revista Extendere. (2016),4(1):76-84.

Silva, J. A. G. D. Estimativa 2014 - Incidência de Câncer no Brasil. (2014).

Souza R. J. S. P., et al. Estimativa do custo do tratamento do câncer de pele tipo não-melanoma no Estado de São Paulo - Brasil. Anais Brasileiros de Dermatologia. 86. 
Research, Society and Development, v. 10, n. 7, e47310717037, 2021

(CC BY 4.0) | ISSN 2525-3409 | DOI: http://dx.doi.org/10.33448/rsd-v10i7.17037

Tuorkey, M. J. Solar ultraviolet radiation from cancer induction to cancer prevention: solar ultraviolet radiation and cell biology. Eur J Cancer Prev. (2015) Sep,24(5):430-8.

Wang, S. Q., Balagula, Y., \& Osterwalder, U. Photoprotection: a review of the current and future technologies. Dermatol Ther 2016.

Wolf, P., Hoffmann, C., Quehenberger, F., Grinschgl, S., \& Kerl, H. Immune protection factors of chemical sunscreens measured in the local contact hypersensitivity model in humans. J Invest Dermatol. (2003),121(5). 